\title{
An elastic hollow cylinder under axial tension containing a crack and two rigid inclusions of ring shape
}

\author{
H. Secil Altundag Artem ${ }^{a, *}$, M. Rusen Gecit ${ }^{b}$ \\ a Department of Mechanical Engineering, Izmir Institute of Technology, Gulbahce Koyu 35437, Urla-Izmir, Turkey \\ ${ }^{\mathrm{b}}$ Department of Engineering Sciences, Middle East Technical University, Ankara 06531, Turkey
}

Received 10 January 2002; accepted 4 July 2002

\begin{abstract}
This paper is concerned with the fracture of an axisymmetric hollow cylindrical bar containing rigid inclusions. The cylinder is under the action of uniformly distributed axial tension applied at infinity. The bar contains a ring-shaped crack at the symmetry plane whose surfaces are free of tractions and two ring-shaped rigid inclusions with negligible thickness symmetrically located on both sides of the crack. It is assumed that the material of the cylinder is linearly elastic and isotropic. The mixed boundary conditions of the problem lead the analysis to a system of three singular integral equations for crack surface displacement derivative and normal and shearing stress jumps on rigid inclusions. These integral equations are solved numerically and the stress intensity factors are calculated.
\end{abstract}

(C) 2002 Civil-Comp Ltd. and Elsevier Science Ltd. All rights reserved.

Keywords: Fracture; Crack; Rigid inclusion; Singular integral equation; Stress intensity factor

\section{Introduction}

Sneddon and Welch [1] analyzed the distribution of stress in a long circular cylinder of elastic material containing a penny-shaped crack at the center of the cylinder. The elastostatic plane problem of a finite strip has been solved by Gecit and Turgut [2]. In this study, solution of the problem is obtained by considering (i) an infinite strip containing a transverse rigid inclusion at the middle and (ii) two symmetrically located transverse cracks. Turgut and Gecit [3] have considered a semiinfinite elastic strip which contains a transverse central crack. Formulation is reduced to a system of three singular integral equations. Gauss-Chebyshev integration formulas for singular integrals are developed by Erdogan and Gupta [4]. Using these formulas a simple numerical method for solving a system of singular integral equations is described in this paper. Gupta [5] considered a semi-infinite strip held rigidly on its short end.

\footnotetext{
${ }^{*}$ Corresponding author.
}

Stress singularity at the strip corner is obtained from the singular integral equation. Stress along the rigid end is determined and the effect of the material properties on the stress-intensity factor is presented. Gupta [6] analyzed the axisymmetric semi-infinite cylinder with fixed short end. Loads are applied far from the fixed end of the cylinder. An integral transform technique is used to formulate the problem in terms of a singular integral equation. This technique has been used by Gupta [5]. Numerous analytical studies have been devoted to the analysis of semi-infinite cylinders with stress-free curved surfaces and prescribed stress or displacement boundary conditions on the plane end. A few good solutions exist. Benthem and Minderhood [7] used the eigenfunction technique to solve semi-infinite and finite cylinder problem with remarkable success. Erdol and Erdogan [8] studied an elastostatic axisymmetric problem for a long thick-walled cylinder containing a ring-shaped internal or edge crack. Using transform technique the problem is formulated in terms of an integral equation which has a simple Cauchy kernel for the internal crack and a generalized Cauchy kernel for the edge crack as the dominant 
part. Delale and Erdogan [9] analyzed the problem of a hollow cylinder which contains an arbitrarily oriented radial crack. The cylinder is subjected to arbitrary normal tractions on the crack surfaces. Problem is formulated in terms of a singular integral equation by using the basic dislocation solutions as the Green's functions. In the paper by Nied and Erdogan [10] the elasticity problem for a long hollow circular cylinder containing an axisymmetric circumferential crack is considered. The cylinder is subjected to general non-axisymmetric external loads. The problem is formulated in terms of a system of singular integral equations with the Fourier coefficients of the derivative of the crack surface displacement density functions. The stress intensity factors and the crack opening displacement are calculated.

The present paper investigates the stress intensity factors for the infinite hollow cylinder containing a ringshaped crack whose surfaces are free of tractions and two ring-shaped rigid inclusions symmetrically located on both sides of the crack with arbitrary, but equal widths. The hollow cylinder is under the action of axisymmetric tensile loads at infinity. Material of the cylinder is assumed to be linearly elastic and isotropic. Solution for this problem can be obtained by superposing the solutions for (1) an infinite cylinder subjected to uniformly distributed tensile load at infinity, and (2) an infinite cylinder having a ring-shaped crack and two rigid inclusions (the perturbation problem). The main objective of this study is to have a good acquaintance with the mathematical difficulties which may arise in a hollow cylinder containing flaws like cracks and rigid inclusions. Afterwards, the problem of a cracked finite cylinder with rigid end plates will be solved. One must be aware that analytical works require a lot of symmetry considerations. Therefore, the geometry of the problem is very much restrictive and one may naturally have concerns on engineering significance and practical applications of the present problem.

\section{Formulation}

Consider the axisymmetric problem for the hollow cylindrical bar shown in Fig. 1. The cylinder with inner and outer radii $A$ and $B$ is subjected to uniformly distributed axial tension of intensity $p_{0}$ at infinity. The infinite hollow cylinder contains a ring-shaped crack of width $(b-a)$ at the symmetry plane $z=0$ and two ringshaped rigid inclusions of width $(d-c)$ at $z= \pm L$ planes.

Along the rigid inclusions with negligible thickness displacements are constant and continuous whereas stresses have jumps. The surfaces of the crack are free of tractions. Therefore, the field equations of axisymmetric elasticity problem must be solved together with the following boundary conditions:

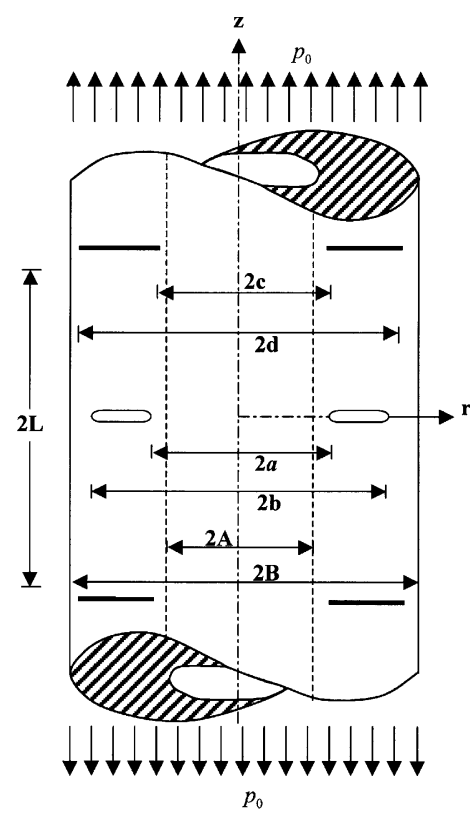

Fig. 1. Geometry of the problem.

$$
\begin{aligned}
& \sigma_{z}(r, 0)=0, \quad a<r<b, \\
& w(r, 0)=0, \quad A<r<a, \quad b<r<B, \\
& \sigma_{z}(r,+\infty)=p_{0}, \quad A<r<B, \\
& \sigma_{z}(r,-\infty)=p_{0}, \quad A<r<B, \\
& u(r,+L)=0, \quad c<r<d, \\
& u(r,-L)=0, \quad c<r<d, \\
& w(r,+L)=\text { const }, \quad c<r<d, \\
& w(r,-L)=\text { const }, \quad c<r<d, \\
& \sigma_{r}(A, z)=0, \quad-\infty<z<\infty, \\
& \tau_{r z}(A, z)=0, \quad-\infty<z<\infty, \\
& \sigma_{r}(B, z)=0, \quad-\infty<z<\infty, \\
& \tau_{r z}(B, z)=0, \quad-\infty<z<\infty,
\end{aligned}
$$

where $u$ and $w$ are the $r$ - and $z$-components of the displacement vector.

Solution for the problem shown in Fig. 1 may be obtained conveniently by considering (1) problem of an infinite hollow cylinder subjected to loads at infinity with no crack or inclusions, and (2) problem of an infinite 


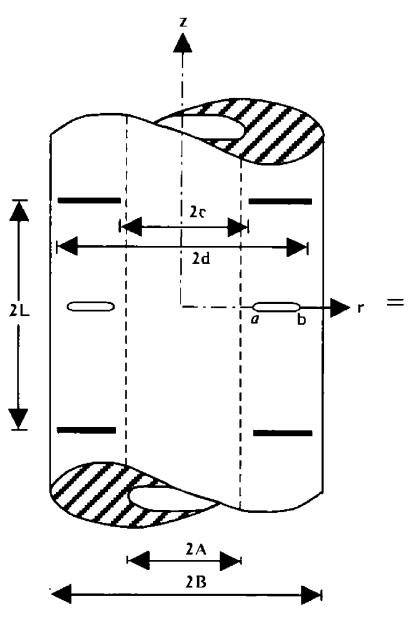

Hankel transform

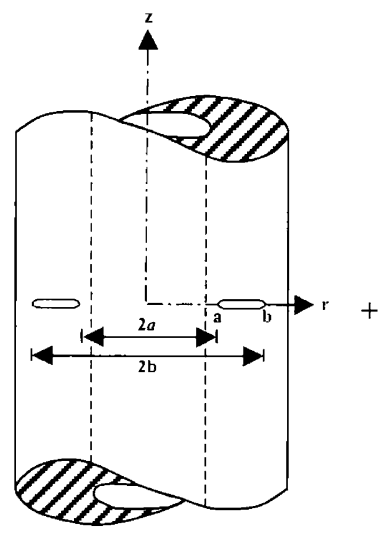

(i)

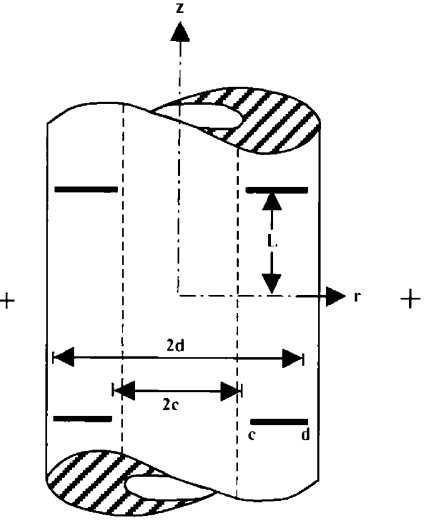

(ii)

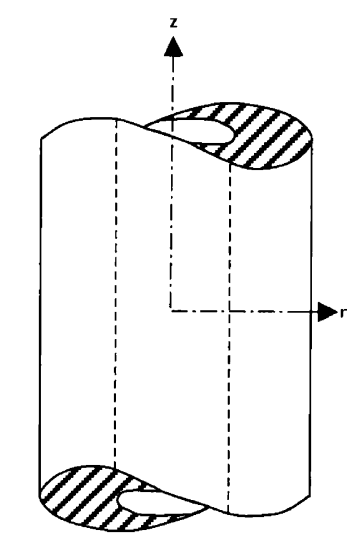

(iii)

Fig. 2. The informal superposition scheme (perturbation problem).

hollow cylinder containing a ring-shaped crack at $z=0$ and two ring-shaped rigid inclusions at $z= \pm L$ shown in Fig. 2. The external load is the negative of the stresses and displacements at locations of the crack and the inclusions obtained from the first problem. Due to symmetry and uniform nature of deformation, the general solution for the displacement and stress components for the first problem is obtained easily in the form

$u(r)=-\frac{(\kappa-3) p_{0}}{2 \mu(\kappa-7)} r$

$w(z)=\frac{-2 p_{0}}{\mu(\kappa-7)} z$,

$\sigma_{r}(r, z)=0$,

$\sigma_{z}(r, z)=p_{0}$

$\tau_{r z}(r, z)=0$,

where $\mu$ is the shear modulus, $\kappa=3-4 v$ as in plane strain problems, $v$ being the Poisson's ratio.

In order that all boundary conditions given in Eqs. (1a)-(5b) be satisfied, the number of unknowns in the expressions for the stress and displacements must be equal to the number of these boundary conditions. Therefore, the general expressions for the stress and displacements for the second problem will be written as the sum of the expressions obtained from the following three subproblems: (i) An infinite axisymmetric elastic medium containing a ring-shaped crack of width $(b-a)$ at $z=0$ plane. Navier equations are solved by the use of the Hankel transform on $r$. Upper and lower half spaces are considered to be two separate media and the general expressions are matched at $z=0$ by means of the following conditions:

$\sigma_{z}\left(r, 0^{+}\right)=\sigma_{z}\left(r, 0^{-}\right), \quad 0 \leqslant r<\infty$,

$\tau_{r z}\left(r, 0^{+}\right)=\tau_{r z}\left(r, 0^{-}\right), \quad 0 \leqslant r<\infty$,

$\frac{\partial}{\partial r}\left[u\left(r, 0^{+}\right)-u\left(r, 0^{-}\right)\right]=0, \quad 0 \leqslant r<\infty$,

$\frac{\partial}{\partial r}\left[w\left(r, 0^{+}\right)-w\left(r, 0^{-}\right)\right]=2 f(r), \quad 0 \leqslant r<\infty$,

where $f(r)$ is the unknown crack surface displacement derivative. Then, the general expressions for the displacements and the stresses in the upper half space become:

$u(r, z)=\frac{1}{\kappa+1} \int_{0}^{\infty}[-2 \alpha z+(\kappa-1)] F(\alpha) \mathrm{e}^{-\alpha z} J_{1}(\alpha r) \mathrm{d} \alpha$

$w(r, z)=\frac{1}{\kappa+1} \int_{0}^{\infty}[-2 \alpha z-(\kappa+1)] F(\alpha) \mathrm{e}^{-\alpha z} J_{0}(\alpha r) \mathrm{d} \alpha$,

$$
\begin{aligned}
\sigma_{r}(r, z)= & \frac{2 \mu}{\kappa+1} \int_{0}^{\infty}[-2 \alpha z(\kappa-1)] F(\alpha) \mathrm{e}^{-\alpha z} \frac{1}{r} J_{1}(\alpha r) \mathrm{d} \alpha \\
& +\frac{2 \mu}{\kappa+1} \int_{0}^{\infty} 2(1-\alpha z) F(\alpha) \mathrm{e}^{-\alpha z} \alpha J_{0}(\alpha r) \mathrm{d} \alpha
\end{aligned}
$$

$\sigma_{z}(r, z)=\frac{4 \mu}{\kappa+1} \int_{0}^{\infty}(\alpha z+1) F(\alpha) \mathrm{e}^{-\alpha z} \alpha J_{0}(\alpha r) \mathrm{d} \alpha$ 
$\tau_{r z}(r, z)=\frac{4 \mu}{\kappa+1} \int_{0}^{\infty} \alpha z F(\alpha) \mathrm{e}^{-\alpha z} \alpha J_{1}(\alpha r) \mathrm{d} \alpha$,

where $J$ is the Bessel function of the first kind and

$F(\alpha)=\int_{a}^{b} f(r) r J_{1}(\alpha r) \mathrm{d} r$

$f(r)=0, \quad A<r<a, \quad b<r<B$

(ii) An infinite axisymmetric elastic medium containing two ring-shaped rigid inclusions of width $(d-c)$ at $z= \pm L$. Navier equations are solved again by the use of the Hankel transform on $r$ satisfying the conditions

$u\left(r, L^{+}\right)=u\left(r, L^{-}\right), \quad A<r<B$,

$w\left(r, L^{+}\right)=w\left(r, L^{-}\right), \quad A<r<B$,

$\sigma_{z}\left(r, L^{+}\right)-\sigma_{z}\left(r, L^{-}\right)=h(r), \quad c<r<d$,

$\tau_{r z}\left(r, L^{+}\right)-\tau_{r z}\left(r, L^{-}\right)=g(r), \quad c<r<d$,

at $z=L$. Here the unknown functions $g(r)$ and $h(r)$, normal and shearing stress jumps along the inclusions, satisfy the conditions

$h(r)=0, \quad A<r<c, d<r<B$,

$g(r)=0 \quad A<r<c, \quad d<r<B$.

Hence, the following expressions may be written for the displacements and the stresses in the interval $(-L \leqslant$ $z \leqslant L$ )

$$
\begin{aligned}
u(r, z)= & \frac{1}{2 \mu(\kappa+1)} \int_{0}^{\infty}[\{-\alpha(L-z) H(\alpha) \\
& +[\alpha(L-z)+\kappa] G(\alpha)\} \mathrm{e}^{-\alpha(z-L)} \\
& +\{-\alpha(L+z) H(\alpha)+[-\alpha(L+z) \\
& \left.+\kappa] G(\alpha)\} \mathrm{e}^{-\alpha(z+L)}\right] J_{1}(\alpha r) \mathrm{d} \alpha,
\end{aligned}
$$

$$
\begin{aligned}
w(r, z)= & \frac{1}{2 \mu(\kappa+1)} \int_{0}^{\infty}[\{[-\alpha(L-z)+\kappa] H(\alpha) \\
& +[\alpha(L-z)] G(\alpha)\} \mathrm{e}^{-\alpha(z-L)}+\{[-\alpha(L+z)-\kappa] H(\alpha) \\
& \left.-\alpha(L+z) G(\alpha)\} \mathrm{e}^{-\alpha(z+L)}\right] \alpha J_{0}(\alpha r) \mathrm{d} \alpha,
\end{aligned}
$$

$$
\begin{aligned}
\sigma_{r}(r, z)= & \frac{1}{2(\kappa+1)} \int_{0}^{\infty}[\{2 \alpha(L-z) H(\alpha)+[-2 \alpha(L-z) \\
& -2 \kappa] G(\alpha)\} \mathrm{e}^{-\alpha(z-L)}+\{2 \alpha(L+z) H(\alpha) \\
& \left.+[2 \alpha(L+z)-2 \kappa] G(\alpha)\} \mathrm{e}^{-\alpha(z+L)}\right] \frac{1}{r} J_{1}(\alpha r) \mathrm{d} \alpha \\
& +\frac{1}{2((\kappa+1))} \int_{0}^{\infty}[\{[-2 \alpha(L-z)+(\kappa-3)] H(\alpha) \\
& +[2 \alpha(L-z)+(\kappa+3)] G(\alpha)\} \mathrm{e}^{-\alpha(z-L)} \\
& +\{[-2 \alpha(L+z)-(\kappa-3)] H(\alpha)+[-2 \alpha(L+z) \\
& \left.+(\kappa+3)] G(\alpha)\} \mathrm{e}^{-\alpha(z+L)}\right] \alpha J_{0}(\alpha r) \mathrm{d} \alpha,
\end{aligned}
$$

$$
\begin{aligned}
\sigma_{z}(r, z)= & \frac{1}{2(\kappa+1)} \int_{0}^{\infty}[\{[2 \alpha(L-z)-(\kappa+1)] H(\alpha) \\
& +[-2 \alpha(L-z)-(\kappa-1)] G(\alpha)\} \mathrm{e}^{-\alpha(z-L)} \\
& +\{[2 \alpha(L+z)-(\kappa+1)] H(\alpha)+[-2 \alpha(L+z) \\
& \left.-(\kappa-1)] G(\alpha)\} \mathrm{e}^{-\alpha(z+L)}\right] \alpha J_{0}(\alpha r) \mathrm{d} \alpha \\
\tau_{r z}(r, z)= & \frac{1}{2(\kappa+1)} \int_{0}^{\infty}[\{[2 \alpha(L-z)-(\kappa-1)] H(\alpha) \\
& +[-2 \alpha(L-z)-(\kappa+1)] G(\alpha)\} \mathrm{e}^{-\alpha(z-L)} \\
& +\{[2 \alpha(L+z)+(\kappa-1)] H(\alpha)+[2 \alpha(L+z) \\
& \left.-(\kappa+1)] G(\alpha)\} \mathrm{e}^{-\alpha(z+L)}\right] \alpha J_{1}(\alpha r) \mathrm{d} \alpha .
\end{aligned}
$$

Here

$G(\alpha)=\int_{c}^{d} g(r) r J_{0}(\alpha r) \mathrm{d} r$

$H(\alpha)=\int_{c}^{d} h(r) r J_{1}(\alpha r) \mathrm{d} r$

(iii) An elastic medium with no crack or inclusion. By using Fourier sine/cosine transforms in $z$-direction the general expressions are obtained as

$$
\begin{aligned}
u(r, z)=\frac{2}{\pi} & \int_{0}^{\infty}\left[-\frac{1}{2} c_{1} I_{1}(\lambda r)+\frac{1}{2} c_{2} K_{1}(\lambda r)\right. \\
& \left.+c_{3} \lambda r I_{0}(\lambda r)+c_{4} \lambda r K_{0}(\lambda r)\right] \cos \lambda z \mathrm{~d} \lambda
\end{aligned}
$$

$$
\begin{aligned}
w(r, z)=\frac{2}{\pi} & \int_{0}^{\infty}\left\{\frac{1}{2} c_{1} I_{0}(\lambda r)+\frac{1}{2} c_{2} K_{0}(\lambda r)\right. \\
& -c_{3}\left[(\kappa+1) I_{0}(\lambda r)+\lambda r I_{1}(\lambda r)\right] \\
& \left.+c_{4}\left[(\kappa+1) K_{0}(\lambda r)-\lambda r K_{1}(\lambda r)\right]\right\} \sin \lambda z \mathrm{~d} \lambda,
\end{aligned}
$$

$$
\begin{aligned}
\sigma_{r}(r, z)=\frac{2 \mu}{\pi} & \int_{0}^{\infty}\left\{c_{1}\left[-\lambda I_{0}(\lambda r)+\frac{1}{r} I_{1}(\lambda r)\right]\right. \\
& +c_{2}\left[-\lambda K_{0}(\lambda r)-\frac{1}{r} K_{1}(\lambda r)\right] \\
& +c_{3}\left[(\kappa-1) \lambda I_{0}(\lambda r)+2 \lambda^{2} r I_{1}(\lambda r)\right] \\
& \left.+c_{4}\left[(\kappa-1) \lambda K_{0}(\lambda r)-2 \lambda^{2} r K_{1}(\lambda r)\right]\right\} \cos \lambda z \mathrm{~d} \lambda,
\end{aligned}
$$

$$
\begin{aligned}
\sigma_{z}(r, z)=\frac{2 \mu}{\pi} & \int_{0}^{\infty}\left\{c_{1} \lambda I_{0}(\lambda r)+c_{2} \lambda K_{0}(\lambda r)\right. \\
& -c_{3}\left[(\kappa+5) \lambda I_{0}(\lambda r)+2 \lambda^{2} r I_{1}(\lambda r)\right] \\
& -c_{4}\left[(\kappa+5) \lambda K_{0}(\lambda r)\right. \\
& \left.\left.-2 \lambda^{2} r K_{1}(\lambda r)\right]\right\} \cos \lambda z \mathrm{~d} \lambda,
\end{aligned}
$$




$$
\begin{aligned}
\tau_{r z}(r, z)=\frac{2 \mu}{\pi} & \int_{0}^{\infty}\left\{c_{1} \lambda I_{1}(\lambda r)-c_{2} \lambda K_{1}(\lambda r)\right. \\
& -c_{3}\left[2 \lambda^{2} r I_{0}(\lambda r)+(\kappa+1) \lambda I_{1}(\lambda r)\right] \\
& -c_{4}\left[2 \lambda^{2} r K_{0}(\lambda r)\right. \\
& \left.\left.-(\kappa+1) \lambda K_{1}(\lambda r)\right]\right\} \sin \lambda z \mathrm{~d} \lambda
\end{aligned}
$$

When the general expressions for these subproblems added together and substituted in (4a), (4b), (5a), and (5b), the stress boundary conditions at inner and outer lateral surfaces of the cylinder, the general expressions for the displacement and stress components will be obtained in terms of three unknown functions $F(\alpha), G(\alpha)$ and $H(\alpha)$.

\section{Integral equations}

The three unknown functions $F(\alpha), G(\alpha)$ and $H(\alpha)$ can be determined by using the remaining boundary conditions (1a), (3a) and (3c) on crack and the inclusions. It is noted that (3a) and (3c) are displacement type conditions whereas (1a) is a stress type condition. In order to have the same type of conditions, say stress type, the boundary conditions may be stated as follows:

$\sigma_{z}(r, 0)=-p_{0}, \quad a<r<b$,

$\frac{\partial}{\partial r} w(r, L)=0, \quad c<r<d$,

$\frac{1}{r} \frac{\partial}{\partial r}[r u(r, L)]=2 \varepsilon_{0}, \quad c<r<d$,

where

$\varepsilon_{0}=\frac{3-\kappa}{7-\kappa} \frac{p_{0}}{2 \mu}$.

Substituting sum of expressions given in (6a), (6b), (7a)(7c), (9a), (9b), (10a)-(10c), (15a)-(15e), (17a)-(17e) for displacements and stresses in (18a)-(18c), separating the divergent integrals giving the simple Cauchy-type singularity [11] after lengthy manipulations, following three singular integral equations are obtained (see [12] for details)

$$
\begin{aligned}
& \frac{2 \mu}{\pi(\kappa+1)} \int_{a}^{b} f(t)\left[\frac{2}{t-r}+2 M_{1}(r, t)+t N_{11}(r, t)\right] \mathrm{d} t \\
& +\frac{1}{\kappa+1} \int_{c}^{d} h(t) t\left[T_{1}(r, t)-\frac{2}{\pi} N_{12}(r, t)\right] \mathrm{d} t \\
& +\frac{1}{\kappa+1} \int_{c}^{d} g(t) t\left[T_{2}(r, t)-\frac{2}{\pi} N_{13}(r, t)\right] \mathrm{d} t=-p_{0}, \\
& a<r<b
\end{aligned}
$$

$$
\begin{aligned}
& \frac{1}{\kappa}+1 \int_{a}^{b} f(t) t\left[T_{3}(r, t)+\frac{2}{\pi} N_{21}(r, t)\right] \mathrm{d} t \\
& \quad+\frac{1}{2 \mu(\kappa+1)} \int_{c}^{d} h(t)\left[t T_{4}(r, t)+\frac{\kappa}{\pi} M_{2}(r, t)\right. \\
& \left.\quad+\frac{\kappa}{\pi} \frac{1}{t-r}-\frac{4}{\pi} t N_{22}(r, t)\right] \mathrm{d} t+\frac{1}{2 \mu(\kappa+1)} \\
& \quad \times \int_{c}^{d} g(t) t\left[T_{5}(r, t)-\frac{4}{\pi} N_{23}(r, t)\right] \mathrm{d} t \\
& =0, \quad c<r<d
\end{aligned}
$$

$$
\begin{aligned}
& \frac{1}{\kappa+1} \int_{a}^{b} f(t) t\left[T_{6}(r, t)+\frac{2}{\pi} N_{31}(r, t)\right] \mathrm{d} t \\
& \quad-\frac{1}{2 \mu(\kappa+1)} \int_{c}^{d} h(t) t\left[T_{7}(r, t)+\frac{4}{\pi} N_{32}(r, t)\right] \mathrm{d} t \\
& \quad+\frac{1}{2 \mu(\kappa+1)} \int_{c}^{d} g(t)\left[t T_{8}(r, t)+\frac{\kappa}{\pi} M_{3}(r, t)\right. \\
& \left.+\frac{\kappa}{\pi} \frac{1}{t-r}-\frac{4}{\pi} t N_{33}(r, t)\right] \mathrm{d} t=2 \varepsilon_{0}, \quad c<r<d
\end{aligned}
$$

where

$M_{i}(r, t)=\frac{M_{i}^{*}(r, t)-1}{t-r}, \quad i=1-3$

and

$M_{1}^{*}(r, t)= \begin{cases}\frac{2(t-r)}{r} K\left(\frac{t}{r}\right)+\frac{2 r}{t+r} E\left(\frac{t}{r}\right), & r>t, \\ \frac{2 t}{t+r} E\left(\frac{r}{t}\right), & r<t,\end{cases}$

$M_{2}^{*}(r, t)= \begin{cases}\frac{2 t}{t+r} E\left(\frac{t}{r}\right), & r>t, \\ -\frac{2}{r}(t-r) K\left(\frac{r}{t}\right)+\frac{2 t^{2} / r}{(t+r)} E\left(\frac{r}{t}\right), & r<t,\end{cases}$

$M_{3}^{*}(r, t)= \begin{cases}\frac{2(t-r)}{r} K\left(\frac{t}{r}\right)+\frac{2 r}{t+r} E\left(\frac{t}{r}\right), & r>t, \\ \frac{2 t}{t+r} E\left(\frac{r}{t}\right), & r<t,\end{cases}$

in which $K$ and $E$ are the complete elliptic integrals of the first and the second kinds, respectively. The kernels $N_{i j}(i, j=1-3)$ are given in Appendix A by (A.1) and the elliptic integrals $T_{i}(r, t)(i=1-8)$ are defined by (A.2). Eqs. (20a)-(20c), must be solved together with the following single-valuedness condition for the displacement around the crack and the equilibrium conditions along the inclusions: 
$\int_{a}^{b} f(t) \mathrm{d} t=0, \quad \int_{c}^{d} g(t) t \mathrm{~d} t=0, \quad \int_{c}^{d} h(t) t \mathrm{~d} t=0$.

The integral equations have (i) a simple Cauchy type singularity at $t=r$, (ii) the kernels $M_{1}, M_{2}, M_{3}$ have logarithmic singularity, (iii) among the Fredholm kernels, $N_{i j}$ only $N_{11}, N_{22}$ and $N_{33}$ have singular terms when $t=A, B$ and $r= \pm A, \pm B$ due to the behaviour of the integrands of the integrals giving $N_{11}, N_{22}$ and $N_{33}$ as $\lambda \rightarrow \infty$. Therefore, $N_{i j}(r, t)$ can be written in the following form:

$N_{i j}(r, t)=\int_{0}^{\infty} L_{i j}(r, t, \lambda) \mathrm{d} \lambda, \quad i, j=1-3$.

Then the singular parts of the kernels may be separated as

$N_{i j s}(r, t)=\int_{0}^{\infty} L_{i j \infty}(r, t, \lambda) \mathrm{d} \lambda, \quad i, j=1-3$,

where

$L_{i j \infty}(r, t, \lambda)=\lim _{\lambda \rightarrow \infty} L_{i j}(r, t, \lambda), \quad i, j=1-3$.

Integrands of integrals given by (24) contain modified Bessel functions. By using asymptotic expansions for modified Bessel functions, the expressions for $L_{i j \infty}(r, t, \lambda)$ are obtained and presented in Appendix (A.1). Corresponding singular kernels $N_{i j s}$ can be obtained in the form

$$
\begin{aligned}
N_{11 s}(r, t)=\frac{1}{\sqrt{r t}} & \left\{\left[-4(B-r)^{2} \frac{\mathrm{d}^{2}}{\mathrm{~d} r^{2}}+12(B-r) \frac{\mathrm{d}}{\mathrm{d} r}-2\right]\right. \\
\times & \frac{1}{(t+r-2 B)}+\left[-4(A-r) \frac{\mathrm{d}^{2}}{\mathrm{~d} r^{2}}\right. \\
& \left.\left.+12(A-r) \frac{\mathrm{d}}{\mathrm{d} r}-2\right] \frac{1}{(t+r-2 A)}\right\},
\end{aligned}
$$

$$
\begin{aligned}
N_{22 s}(r, t)=\frac{1}{\sqrt{r t}} & \left\{\left[-(B-r)^{2} \frac{\mathrm{d}^{2}}{\mathrm{~d} r^{2}}+3(B-r) \frac{\mathrm{d}}{\mathrm{d} r}\right.\right. \\
+ & \left.\frac{1}{4}\left(\kappa^{2}-3\right)\right] \frac{1}{(t+r-2 B)} \\
+ & {\left[-(A-r)^{2} \frac{\mathrm{d}^{2}}{\mathrm{~d} r^{2}}+3(A-r) \frac{\mathrm{d}}{\mathrm{d} r}\right.} \\
+ & \left.\left.\frac{1}{4}\left(\kappa^{2}-3\right)\right] \frac{1}{(t+r-2 A)}\right\},
\end{aligned}
$$

$$
\begin{aligned}
N_{33 s}(r, t)=\frac{1}{\sqrt{r} t} & \left\{\left[-(B-r)^{2} \frac{\mathrm{d}^{2}}{\mathrm{~d} r^{2}}+3(B-r) \frac{\mathrm{d}}{\mathrm{d} r}\right.\right. \\
& \left.+\frac{1}{4}\left(\kappa^{2}-3\right)\right] \frac{1}{(t+r-2 B)} \\
+ & {\left[-(A-r)^{2} \frac{\mathrm{d}^{2}}{\mathrm{~d} r^{2}}+3(A-r) \frac{\mathrm{d}}{\mathrm{d} r}\right.} \\
+ & \left.\left.\frac{1}{4}\left(\kappa^{2}-3\right)\right] \frac{1}{(t+r-2 A)}\right\},
\end{aligned}
$$

and the bounded parts of the kernels will be

$$
\begin{gathered}
N_{i j b}(r, t)=\int_{0}^{\infty}\left[L_{i j}(r, t, \lambda)-L_{i j \infty}(r, t, \lambda)\right] \mathrm{d} \lambda, \\
i, j=1-3 .
\end{gathered}
$$

Together with $1 /(t-r), N_{11 s}, N_{22 s}, N_{33 s}$ give generalized Cauchy kernels. The singular behaviour of the unknown functions $f(t), g(t)$ and $h(t)$ are determined by writing

$f(t)=F(t)[(t-a)(b-t)]^{-\gamma} \quad 0<\operatorname{Re}(\gamma)<1$,

$h(t)=H(t)[(t-c)(d-t)]^{-\delta} \quad 0<\operatorname{Re}(\delta)<1$,

$g(t)=G(t)[(t-c)(d-t)]^{-\delta} \quad 0<\operatorname{Re}(\delta)<1$,

where $F(t), H(t)$ and $G(t)$ are Hölder-continuous functions in respective intervals $[a, b]$ and $[c, d] . f(t)$ has integrable singularity at the edges of the crack while $g(t)$ and $h(t)$ have at the edges of the inclusions. After somewhat routine manipulations (see [12]) it can be shown that $\gamma$ and $\delta$ satisfy the characteristic equations

$\cot \pi \gamma=0$

$\cot \pi \delta=0$

Therefore $\gamma=\delta=1 / 2$ which is in perfect agreement with previously reported results for the power of singularity at the edges of the crack $(r \rightarrow a, b)$ and at the edges of the inclusions $(r \rightarrow c, d)$.

Having determined the singular behaviour of the unknown functions, the integrals appearing in Eqs. (20a)-(20c) and (23) may be non-dimensionalized by introducing the following dimensionless variables $\tau, \xi$ for the crack by

$t=\frac{b-a}{2} \tau+\frac{b+a}{2}, \quad a<t<b,-1<\tau<1$,

$r=\frac{b-a}{2} \xi+\frac{b+a}{2}, \quad a<r<b,-1<\xi<1$

and $\eta, \varepsilon$ for the inclusions by

$$
\begin{aligned}
& t=\frac{d-c}{2} \eta+\frac{d+c}{2}, \quad c<t<d,-1<\eta<1, \\
& r=\frac{d-c}{2} \varepsilon+\frac{d+c}{2}, \quad c<r<d, \quad-1<\varepsilon<1
\end{aligned}
$$

and Eqs. (20a)-(20c) become

$$
\begin{aligned}
& \frac{1}{\pi} \int_{-1}^{1} \bar{f}(\tau)\left[\frac{2}{\tau-\xi} \widehat{M}_{1}(\xi, \tau)+\widehat{N}_{11}(\xi, \tau)\right] \mathrm{d} \tau \\
& \quad+\frac{p_{0}}{2 \mu} \frac{1}{\pi} \int_{-1}^{1} \bar{h}(\eta)\left[\widehat{T}_{1}(\xi, \eta)-\widehat{N}_{12}(\xi, \eta)\right] \mathrm{d} \eta \\
& \quad+\frac{p_{0}}{2 \mu} \frac{1}{\pi} \int_{-1}^{1} \bar{g}(\eta)\left[\widehat{T}_{2}(\xi, \eta)-\widehat{N}_{13}(\eta, \xi)\right] \mathrm{d} \eta \\
& \quad=-\frac{p_{0}}{2 \mu}(\kappa+1), \quad-1<\xi<1,
\end{aligned}
$$




$$
\begin{aligned}
& \frac{1}{\pi} \int_{-1}^{1} \bar{f}(\tau)\left[\widehat{T}_{3}(\varepsilon, \tau)+\widehat{N}_{21}(\varepsilon, \tau)\right] \mathrm{d} \tau+\frac{p_{0}}{2 \mu} \frac{1}{\pi} \int_{-1}^{1} \bar{h}(\eta) \\
& \quad \times\left[\widehat{T}_{4}(\varepsilon, \eta)+\widehat{M}_{3}(\varepsilon, \eta)+\frac{\kappa}{\eta-\varepsilon}-\widehat{N}_{22}(\varepsilon, \eta)\right] \mathrm{d} \eta \\
& \quad+\frac{p_{0}}{2 \mu} \frac{1}{\pi} \int_{-1}^{1} \bar{g}(\eta)\left[\widehat{T}_{5}(\varepsilon, \eta)-\widehat{N}_{23}(\varepsilon, \eta)\right] \mathrm{d} \eta \\
& \quad=0, \quad-1<\varepsilon<1,
\end{aligned}
$$

$$
\begin{aligned}
& \frac{1}{\pi} \int_{-1}^{1} \bar{f}(\tau)\left[\widehat{T}_{6}(\varepsilon, \tau)+\widehat{N}_{31}(\varepsilon, \tau)\right] \mathrm{d} \tau-\frac{p_{0}}{2 \mu} \frac{1}{\pi} \int_{-1}^{1} \bar{h}(\eta) \\
& \quad \times\left[\widehat{T}_{7}(\varepsilon, \eta)+\widehat{N}_{32}(\varepsilon, \eta)\right] \mathrm{d} \eta+\frac{p_{0}}{2 \mu} \frac{1}{\pi} \int_{-1}^{1} \bar{g}(\eta) \\
& \quad \times\left[\widehat{T}_{8}(\varepsilon, \eta)+\widehat{M}_{3}(\varepsilon, \eta)+\frac{\kappa}{\eta-\varepsilon}-\widehat{N}_{33}(\varepsilon, \eta)\right] \mathrm{d} \eta \\
& \quad=\frac{2(3-\kappa)}{(7-\kappa)}(\kappa+1) \frac{p_{0}}{2 \mu}, \quad-1<\varepsilon<1,
\end{aligned}
$$

$$
\begin{aligned}
& k_{2 c}=\lim _{r \rightarrow c} \sqrt{2(r-c)} \tau_{r z}(r, L), \\
& k_{1 d}=\lim _{r \rightarrow d} \sqrt{2(d-r)} \sigma_{z}(r, L), \\
& k_{2 d}=\lim _{r \rightarrow d} \sqrt{2(d-r)} \tau_{r z}(r, L) .
\end{aligned}
$$

\section{Results}

In the numerical calculations Poisson's ratio is used as the material parameter and the ratios of the outer radius of the cylinder, outer and inner radii of the crack and the inclusions, and the distance between the inclusions and the crack to the inner radius of the cylinder are used as the geometric parameters. Some of the calculated results are presented in graphical form in Figs. 3-8. Figs. 3-5 show the normalized stress intensity factors at the edges of the crack defined by

$\bar{f}(\tau)=f\left(\frac{b-a}{2} \tau+\frac{b+a}{2}\right)$,

$\bar{h}(\eta)=h\left(\frac{d-c}{2} \eta+\frac{d+c}{2}\right)$,

$\bar{g}(\eta)=g\left(\frac{d-c}{2} \eta+\frac{d+c}{2}\right)$.

Then, substituting singular behaviour of the dimensionless unknown functions (Eqs. (29a)-(29c))

$$
\begin{aligned}
& \bar{f}(\tau)=\bar{F}(\tau)\left(1-\tau^{2}\right)^{-1 / 2}, \quad-1<\tau<1, \\
& \bar{h}(\eta)=\bar{H}(\eta)\left(1-\eta^{2}\right)^{-1 / 2}, \quad-1<\eta<1, \\
& \bar{g}(\eta)=\bar{G}(\eta)\left(1-\eta^{2}\right)^{-1 / 2}, \quad-1<\eta<1,
\end{aligned}
$$

these integrals are converted into series by the use of the Gauss-Lobatto integration formula [13] so that a system of linear algebraic equations is obtained. This system is solved numerically. The infinite integrals are calculated using Laguerre and Filon integration formulas [13]. After the numerical solution is obtained, the mode I stress intensity factors at the edges of the crack, $k_{1 a}, k_{1 b}$, the mode I stress intensity factors, $k_{1 c}, k_{1 d}$, and the mode II stress intensity factors, $k_{2 c}, k_{2 d}$, at the edges of the rigid inclusions are calculated:

$$
\begin{aligned}
& k_{1 a}=\lim _{r \rightarrow a} \sqrt{2(a-r)} \sigma_{z}(r, 0), \\
& k_{1 b}=\lim _{r \rightarrow b} \sqrt{2(r-b)} \sigma_{z}(r, 0), \\
& k_{1 c}=\lim _{r \rightarrow c} \sqrt{2(r-c)} \sigma_{z}(r, L),
\end{aligned}
$$

$\bar{k}_{1 a}=k_{1 a} / p_{0} \sqrt{(b-a) / 2}$,

$\bar{k}_{1 b}=k_{1 b} / p_{0} \sqrt{(b-a) / 2}$.

As may be observed from these figures $\bar{k}_{1 a}$ increases when the crack width increases, the inclusion width, and $v$ decrease. On the other hand, $\bar{k}_{1 b}$ increases when the crack width, the inclusion width, and $v$ increase. $\bar{k}_{1 a}$ is

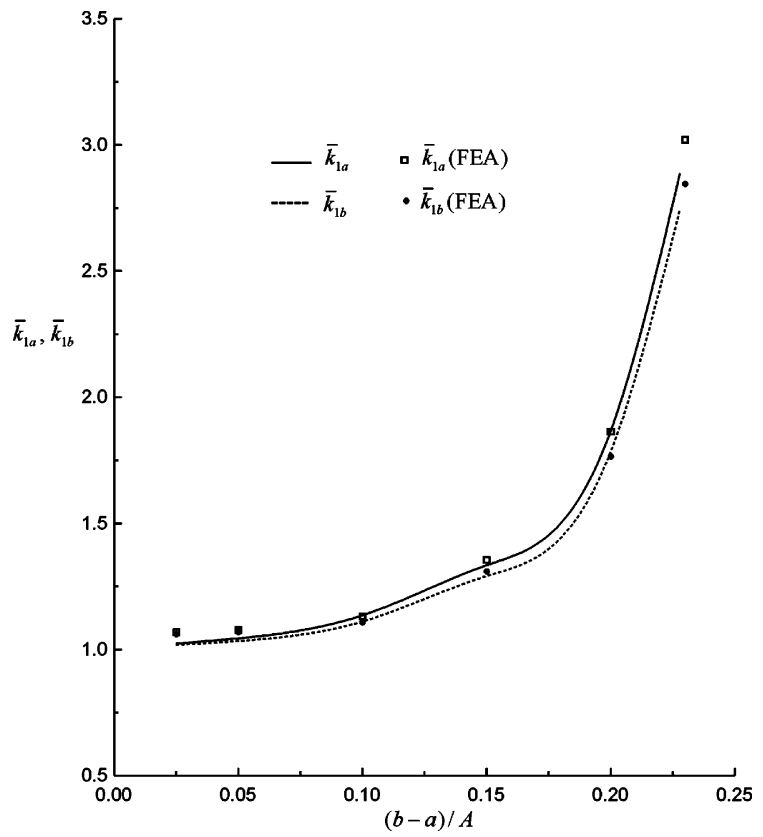

Fig. 3. Variation of normalized stress intensity factors, $\bar{k}_{1 a}, \bar{k}_{1 b}$, with $(b-a) / A$ when $B=1.25 A, L=0.25 A, d-c=0.15 A$, $v=0.3, a+b=2.25 A, c+d=2.25 A$. 


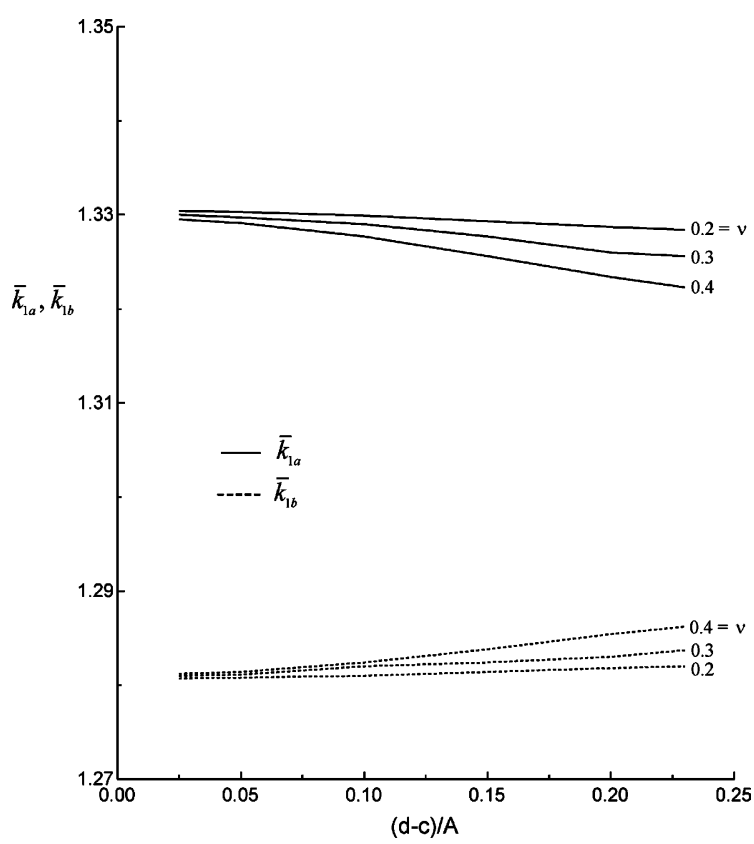

Fig. 4. Variation of normalized stress intensity factors, $\bar{k}_{1 a}, \bar{k}_{1 b}$, with $(d-c) / A$ when $B=1.25 A, L=0.5 A, \quad b-a=0.15 A$, $a+b=2.25 A, c+d=2.25 A$.

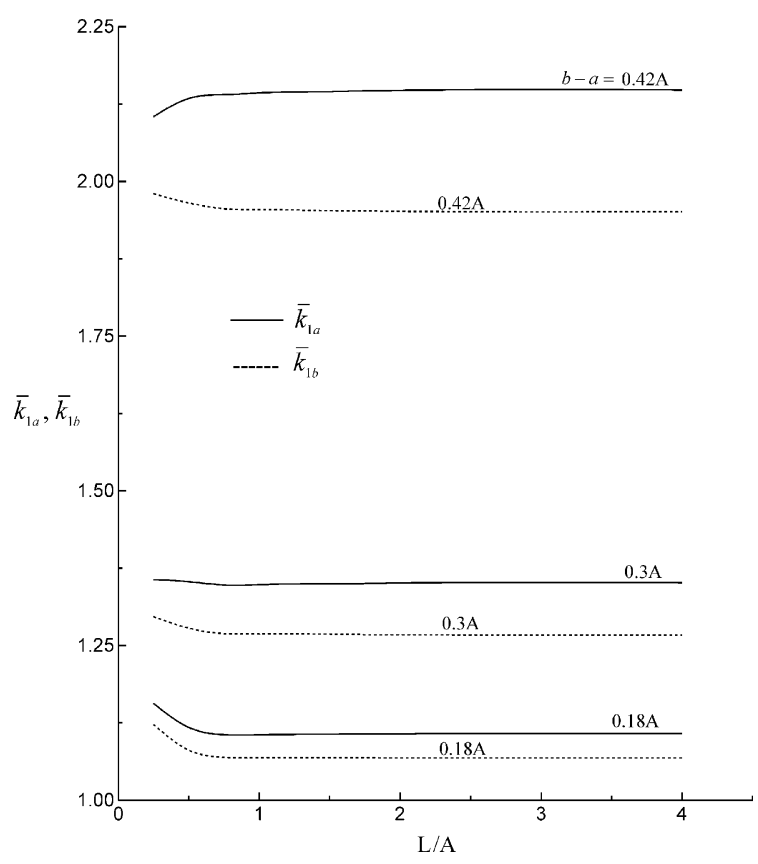

Fig. 5. Variation of normalized stress intensity factors, $\bar{k}_{1 a}, \bar{k}_{1 b}$ with $L / A$ when $B=1.5 A, d-c=0.3 A, v=0.3, a+b=2.5 A$, $c+d=2.5 A$.

greater than $\bar{k}_{1 b}$, both are constant when $L / A \gtrsim 1.0$. In Fig. 3, together with the results of the present study,

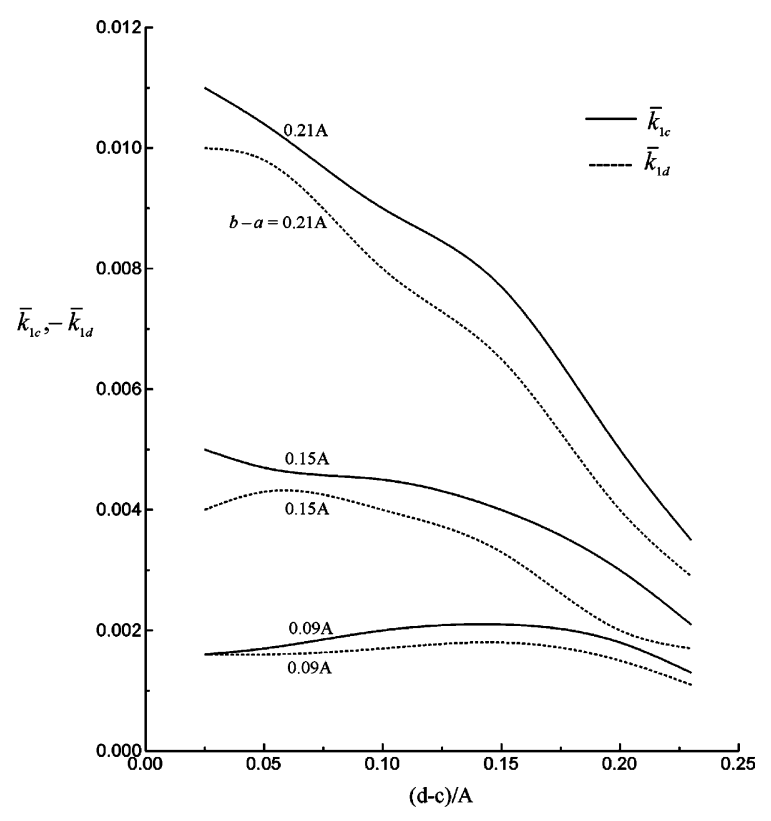

Fig. 6. Variation of normalized stress intensity factors, $\bar{k}_{1 c}, \bar{k}_{1 d}$, with $(d-c) / A$ when $B=1.25 A, L=0.5 A, v=0.3, a+b=$ $2.25 \mathrm{~A}, c+d=2.25 \mathrm{~A}$.

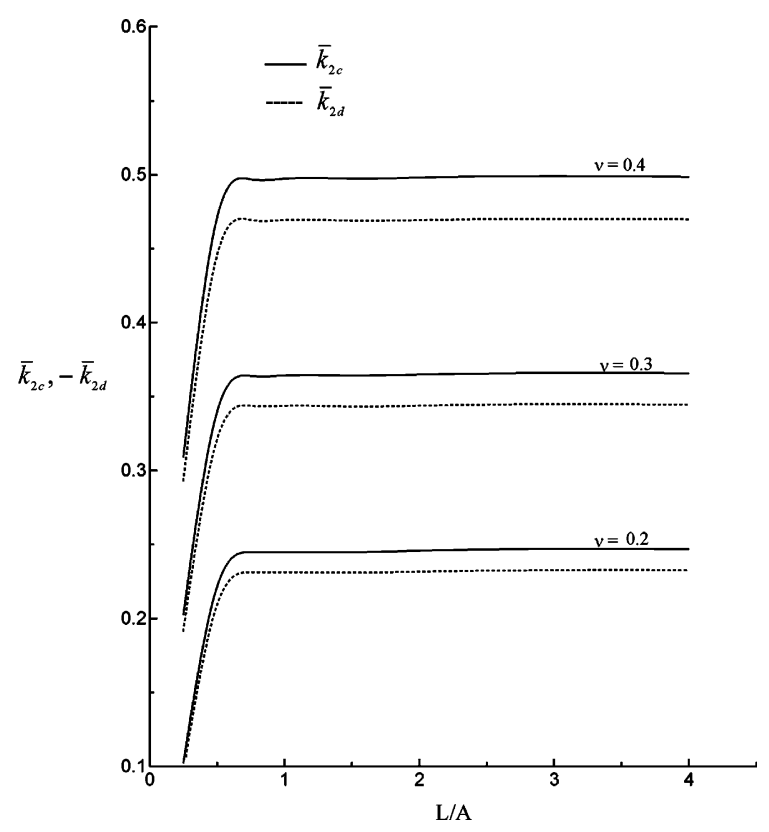

Fig. 7. Variation of normalized stress intensity factors, $\bar{k}_{2 c}, \bar{k}_{2 d}$ with $L / A$ when $B=1.5 A, b-a=0.3 A, d-c=0.1 A, a+b=$ $2.5 A, c+d=2.5 A$.

finite element solution is also presented for comparison purposes. As can be observed in this figure, analytical 


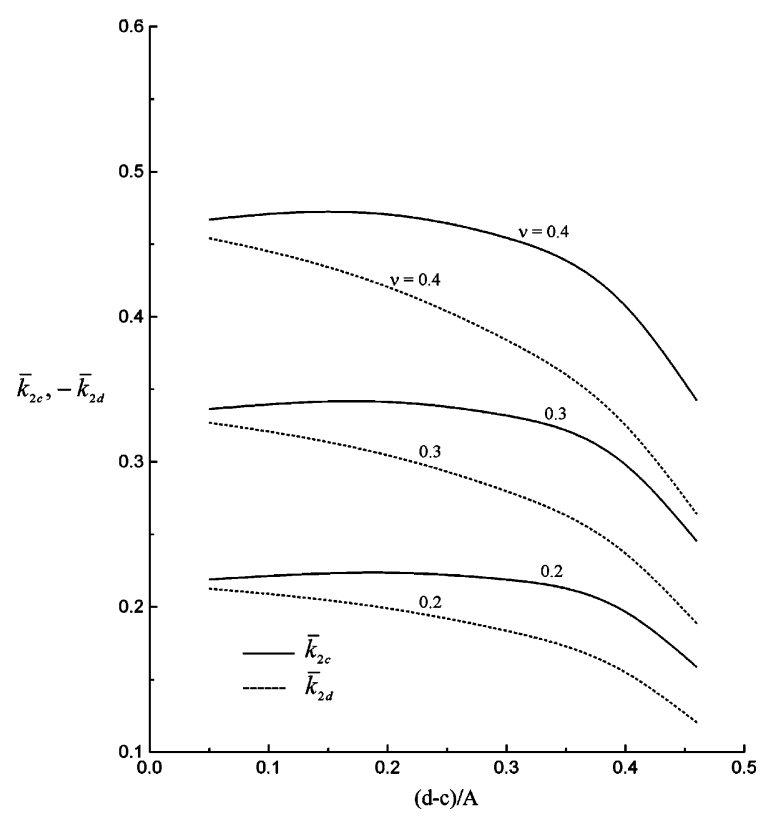

Fig. 8. Variation of normalized stress intensity factors $\bar{k}_{2 c}, \bar{k}_{2 d}$ with $(d-c) / A$ when $B=1.5 A, L=0.5 A, b-a=0.3 A, a+b=$ $2.5 A, c+d=2.5 A$.

results and numerical finite element results are very much similar, coinciding especially for considerable crack lengths. Figs. 6-8 show the normalized stress intensity factors at the edges of the inclusion

$\bar{k}_{1 c}=k_{1 c} / p_{0} \sqrt{(d-c) / 2}$,

$\bar{k}_{1 d}=k_{1 d} / p_{0} \sqrt{(d-c) / 2}$,

$\bar{k}_{2 c}=k_{2 c} / p_{0} \sqrt{(d-c) / 2}$,

$\bar{k}_{2 d}=k_{2 d} / p_{0} \sqrt{(d-c) / 2}$.

It can be observed that $\bar{k}_{1 c}$ and $\bar{k}_{1 d}$ increase as the crack width increases and the inclusion width decreases. $\bar{k}_{2 c}$ and $\bar{k}_{2 d}$ increase as $v$ and $L / A$ increase and as the inclusion width decreases.

\section{Appendix A}

The kernels $N_{i j}(r, t)(i, j=1-3)$ appearing in Eqs. (20a)-(20c) are defined as follows:

$$
\begin{aligned}
N_{i j}(r, t)= & \int_{0}^{\infty} L_{i j}(r, t, \lambda) \mathrm{d} \lambda \\
= & \int_{0}^{\infty} L_{i j \infty}(r, t, \lambda) \mathrm{d} \lambda+\int_{0}^{\infty}\left[L_{i j}(r, t, \lambda)\right. \\
& \left.\quad-L_{i j \infty}(r, t, \lambda)\right] \mathrm{d} \lambda, \quad i, j=1-3,
\end{aligned}
$$

where $L_{i j \infty}(r, t, \lambda)$ may be obtained in the form

$$
\begin{aligned}
L_{11 \infty}(r, t, \lambda)= & \frac{1}{\sqrt{r t}}\left\{\mathrm { e } ^ { - \lambda ( 2 B - r - t ) } \left[-4(B-r)(B-t) \lambda^{2}\right.\right. \\
& +2(B-r) \lambda+6(B-t) \lambda-4]\} \\
& +\frac{1}{\sqrt{r t}}\left\{\mathrm { e } ^ { \lambda ( 2 A - r - t ) } \left[4(A-r)(A-t) \lambda^{2}\right.\right. \\
& +2(A-r) \lambda+6(A-t) \lambda+4]\}, \\
L_{12 \infty}(r, t, \lambda)= & \frac{\sin \lambda L}{\sqrt{r t}}\left\{\mathrm { e } ^ { - \lambda ( 2 B - r - t ) } \left[-2(B-r)(B-t) \lambda^{2}\right.\right. \\
& \left.\left.+\kappa(B-r) \lambda+3(B-t) \lambda-\frac{1}{2}(3 \kappa+1)\right]\right\} \\
& +\frac{\sin \lambda L}{\sqrt{r t}}\left\{\mathrm { e } ^ { \lambda ( 2 A - r - t ) } \left[-2(A-r)(A-t) \lambda^{2}\right.\right. \\
& \left.\left.-\kappa(A-r) \lambda-3(A-t) \lambda-\frac{1}{2}(3 \kappa+1)\right]\right\},
\end{aligned}
$$

$$
\begin{aligned}
L_{13 \infty}(r, t, \lambda)= & \frac{\cos \lambda L}{\sqrt{r t}}\left\{\mathrm { e } ^ { - \lambda ( 2 B - r - t ) } \left[2(B-r)(B-t) \lambda^{2}\right.\right. \\
& \left.\left.+\kappa(B-r) \lambda-3(B-t) \lambda-\frac{1}{2}(3 \kappa-1)\right]\right\} \\
& +\frac{\cos \lambda L}{\sqrt{r t}}\left\{\mathrm { e } ^ { \lambda ( 2 A - r - t ) } \left[-2(A-r)(A-t) \lambda^{2}\right.\right. \\
& \left.\left.+\kappa(A-r) \lambda-3(A-t) \lambda+\frac{1}{2}(3 \kappa-1)\right]\right\},
\end{aligned}
$$

$$
\begin{aligned}
L_{21 \infty}(r, t, \lambda)= & \frac{1}{\sqrt{r t}}\left\{\mathrm { e } ^ { - \lambda ( 2 B - r - t ) } \left[-2(B-r)(B-t) \lambda^{2}\right.\right. \\
& \left.\left.+(B-r) \lambda+(\kappa+2)(B-t) \lambda-\frac{1}{2}(\kappa+3)\right]\right\} \\
& +\frac{1}{\sqrt{r t}}\left\{\mathrm { e } ^ { \lambda ( 2 A - r - t ) } \left[-2(A-r)(A-t) \lambda^{2}\right.\right. \\
& \left.\left.+(A-r) \lambda-(\kappa+2)(A-t) \lambda-\frac{1}{2}(\kappa+3)\right]\right\},
\end{aligned}
$$

$$
\begin{aligned}
L_{22 \infty}(r, t, \lambda)= & \frac{\sin \lambda L}{\sqrt{r t}}\left\{\mathrm { e } ^ { - \lambda ( 2 B - r - t ) } \left[-(B-r)(B-t) \lambda^{2}\right.\right. \\
& +\frac{\kappa}{2}(B-r) \lambda+\left(\frac{\kappa}{2}+1\right)(B-t) \lambda-\frac{1}{4}(\kappa \\
& \left.\left.+1)^{2}\right]\right\}+\frac{\sin \lambda L}{\sqrt{r t}}\left\{\mathrm{e}^{\lambda(2 A-r-t)}[(A-r)(A\right. \\
& -t) \lambda^{2}-\frac{\kappa}{2}(A-r) \lambda-\left(\frac{\kappa}{2}+1\right)(A-t) \lambda \\
& \left.\left.+\frac{1}{4}(\kappa+1)^{2}\right]\right\},
\end{aligned}
$$




$$
\begin{aligned}
L_{23 \infty}(r, t, \lambda)= & \frac{\cos \lambda L}{\sqrt{r t}}\left\{\mathrm { e } ^ { - \lambda ( 2 B - r - t ) } \left[(B-r)(B-t) \lambda^{2}\right.\right. \\
& +\frac{\kappa}{2}(B-r) \lambda-\left(\frac{\kappa}{2}+1\right)(B-t) \lambda \\
& \left.\left.-\frac{1}{4}\left(\kappa^{2}+2 \kappa-1\right)\right]\right\}+\frac{\cos \lambda L}{\sqrt{r t}}\left\{\mathrm{e}^{\lambda(2 A-r-t)}\right. \\
& \times\left[(A-r)(A-t) \lambda^{2}-\frac{\kappa}{2}(A-r) \lambda\right. \\
& \left.\left.+\left(\frac{\kappa}{2}+1\right)(A-t) \lambda-\frac{1}{4}\left(\kappa^{2}+2 \kappa-1\right)\right]\right\},
\end{aligned}
$$

$$
\begin{aligned}
L_{31 \infty}(r, t, \lambda)= & \frac{1}{\sqrt{r t}}\left\{\mathrm { e } ^ { - \lambda ( 2 B - r - t ) } \left[2(B-r)(B-t) \lambda^{2}\right.\right. \\
& \left.\left.-(B-r) \lambda+(\kappa-2)(B-t) \lambda-\frac{1}{2}(\kappa-3)\right]\right\} \\
& +\frac{1}{\sqrt{r t}}\left\{\mathrm { e } ^ { \lambda ( 2 A - r - t ) } \left[-2(A-r)(A-t) \lambda^{2}\right.\right. \\
& -(A-r) \lambda+(\kappa-2)(A-t) \lambda \\
& \left.\left.+\frac{1}{2}(\kappa-3)\right]\right\},
\end{aligned}
$$

$$
\begin{aligned}
L_{32 \infty}(r, t, \lambda)= & \frac{\sin \lambda L}{\sqrt{r t}}\left\{\mathrm { e } ^ { - \lambda ( 2 B - r - t ) } \left[-(B-r)(B-t) \lambda^{2}\right.\right. \\
& -\frac{\kappa}{2}(B-r) \lambda+\frac{1}{2}(\kappa-2)(B-t) \lambda \\
& \left.\left.-\frac{1}{4}\left(\kappa^{2}-2 \kappa-1\right)\right]\right\}+\frac{\sin \lambda L}{\sqrt{r t}}\left\{\mathrm{e}^{\lambda(2 A-r-t)}\right. \\
& \times\left[(A-r)(A-t) \lambda^{2}+\frac{\kappa}{2}(A-r) \lambda\right. \\
& \left.\left.-\frac{1}{2}(\kappa-2)(A-t) \lambda-\frac{1}{4}\left(\kappa^{2}-2 \kappa-1\right)^{2}\right]\right\},
\end{aligned}
$$

$$
\begin{aligned}
L_{33 \infty}(r, t, \lambda)= & \frac{\cos \lambda L}{\sqrt{r t}}\left\{\mathrm { e } ^ { - \lambda ( 2 B - r - t ) } \left[-(B-r)(B-t) \lambda^{2}\right.\right. \\
& -\frac{\kappa}{2}(B-r) \lambda+\frac{1}{2}(\kappa-2)(B-t) \lambda \\
& \left.\left.-\frac{1}{4}(\kappa-1)^{2}\right]\right\}+\frac{\cos \lambda L}{\sqrt{r t}}\left\{\mathrm{e}^{\lambda(2 A-r-t)}\right. \\
& \times\left[(A-r)(A-t) \lambda^{2}+\frac{\kappa}{2}(A-r) \lambda\right. \\
& \left.\left.-\frac{1}{2}(\kappa-2)(A-t) \lambda+\frac{1}{4}(\kappa-1)^{2}\right]\right\} .
\end{aligned}
$$

Expressions of the functions used in Eqs. (20a)-(20c):

$$
\begin{aligned}
& T_{1}(r, t)=2 L k_{21}(r, t)+(\kappa+1) k_{22}(r, t), \\
& T_{2}(r, t)=2 L k_{23}(r, t)-(\kappa-1) k_{22}(r, t), \\
& T_{3}(r, t)=2 L k_{11}(r, t)+(\kappa+1) k_{12}(r, t), \\
& T_{4}(r, t)=2 L k_{13}(r, t)+\kappa k_{14}(r, t), \\
& T_{5}(r, t)=2 L k_{15}(r, t), \\
& T_{6}(r, t)=-2 L k_{36}(r, t)+(\kappa-1) k_{37}(r, t), \\
& T_{7}(r, t)=2 L k_{18}(r, t), \\
& T_{8}(r, t)=-2 L k_{39}(r, t)+\kappa k_{30}(r, t),
\end{aligned}
$$

where

$k_{11}(r, t)=\int_{0}^{\infty} \mathrm{e}^{-\alpha L} \alpha^{2} J_{1}(t \alpha) J_{1}(r \alpha) \mathrm{d} \alpha$,

$k_{12}(r, t)=\int_{0}^{\infty} \mathrm{e}^{-\alpha L} \alpha J_{1}(t \alpha) J_{1}(r \alpha) \mathrm{d} \alpha$,

$k_{13}(r, t)=\int_{0}^{\infty} \mathrm{e}^{-2 L \alpha} \alpha^{2} J_{0}(t \alpha) J_{1}(r \alpha) \mathrm{d} \alpha$

$k_{14}(r, t)=\int_{0}^{\infty} \mathrm{e}^{-2 L \alpha} \alpha J_{0}(t \alpha) J_{1}(r \alpha) \mathrm{d} \alpha$,

$k_{15}(r, t)=\int_{0}^{\infty} \mathrm{e}^{-2 L \alpha} \alpha^{2} J_{1}(t \alpha) J_{1}(r \alpha) \mathrm{d} \alpha$

$k_{21}(r, t)=\int_{0}^{\infty} \mathrm{e}^{-\alpha L} \alpha^{2} J_{0}(t \alpha) J_{0}(r \alpha) \mathrm{d} \alpha$,

$k_{22}(r, t)=\int_{0}^{\infty} \mathrm{e}^{-\alpha L} \alpha J_{0}(t \alpha) J_{0}(r \alpha) \mathrm{d} \alpha$,

$k_{23}(r, t)=\int_{0}^{\infty} \mathrm{e}^{-\alpha L} \alpha^{2} J_{1}(t \alpha) J_{0}(r \alpha) \mathrm{d} \alpha=k_{36}(r, t)$,

$k_{24}(r, t)=\int_{0}^{\infty} \mathrm{e}^{-\alpha L} \alpha J_{1}(t \alpha) J_{0}(r \alpha) \mathrm{d} \alpha=k_{37}(r, t)$,

$k_{38}(r, t)=\int_{0}^{\infty} \mathrm{e}^{-2 \alpha L} \alpha^{2} J_{0}(t \alpha) J_{0}(r \alpha) \mathrm{d} \alpha$,

$k_{39}(r, t)=\int_{0}^{\infty} \mathrm{e}^{-2 \alpha L} \alpha^{2} J_{1}(t \alpha) J_{0}(r \alpha) \mathrm{d} \alpha$,

$k_{30}(r, t)=\int_{0}^{\infty} \mathrm{e}^{-2 \alpha L} \alpha J_{1}(t \alpha) J_{0}(r \alpha) \mathrm{d} \alpha$.

\section{References}

[1] Sneddon IN, Welch JT. A note on the distribution of stress in a cylinder containing penny- shaped crack. Int $\mathbf{J}$ Eng Sci 1963;1:411-9. 
[2] Gecit MR, Turgut A. Extension of a finite strip bonded to a rigid support. Computat Mech 1988;3:398-410.

[3] Turgut A, Gecit MR. A semi-infinite elastic strip containing a transverse crack. The Arabian J Sci Eng 1988;13:7180 .

[4] Erdogan F, Gupta GD. On the numerical solution of singular integral equations. Quart Appl Math 1972;30:52534.

[5] Gupta GD. An integral approach to the semi-infinite strip problem. J Appl Mech 1973;40:948-54.

[6] Gupta GD. The analysis of the semi-infinite cylinder problem. Int J Solids Struct 1974;10:137-48.

[7] Benthem JP, Minderhoud P. The problem of the solid cylinder compressed between rough rigid stamps. Int $\mathbf{J}$ Solids Struct 1972;8:1027-42.
[8] Erdol R, Erdogan F. A thick-walled cylinder with an axisymmetric internal or edge crack. J Appl Mech 1978;45: 281-6.

[9] Delale F, Erdogan F. Stress intensity factors in hollow cylinder containing a radial crack. Int J Fract 1982;20:251-65.

[10] Nied HF, Erdogan F. The elasticity problem for a thickwalled cylinder containing a circumferential crack. Int J Fract 1983;22:277-301.

[11] Muskhelishvili NI. Singular integral equations. Gröningen, Holland: P. Noordhoff; 1953.

[12] Artem H. Problem of cracked infinite hollow cylinder with two rigid inclusions. PhD thesis, Middle East Technical University, Ankara, Turkey, 1999.

[13] Stroud AH, Secrest D. Gaussian quadrature formulas. New York: Prentice-Hall; 1966. 